\title{
Comparative Performance of SDD-EDS and WDS Detectors for Quantitative Analysis of Mineral Specimens: The Next Generation Electron Microprobe
}

\author{
John T. Armstrong ${ }^{1}$ \\ ${ }^{1}$ Geophysical Laboratory, Carnegie Institution of Washington, Washington, DC, USA.
}

With the development of silicon drift detectors (SDD), energy dispersive x-ray emission spectrometry is now capable of making compositional measurements of comparable precision as those that have been routinely obtained by wavelength dispersive spectrometers on electron microprobes [e.g., 1]. Large area silicon drift detectors, when placed near the specimen, subtend significantly larger solid angles than WDS detectors and so can make these measurements at considerably lower beam currents, making them particularly well-suited for use at the conditions ordinarily employed for scanning electron microscopes. The significantly lower resolution of SDDs compared to WDS spectrometers (although significantly improved over $\mathrm{Si}(\mathrm{Li})$ EDS detectors, particularly at low energies) limits their ability to make high precision measurements of minor element concentrations and precludes their determination of trace element concentrations. Additionally there are a number of severe x-ray peak overlaps that require a higher degree of energy/wavelength resolution than is possible with SDDs, despite the high quality of available EDS spectral deconvolution software. For these cases, WDS spectrometers are demonstrably superior in performance.

For the past several years, our laboratory has performed extensive full-standards-based analyses of mineral specimens with Oxford X-Max $70 \mathrm{~mm}^{2}$ detectors on two SEMs. As part of our quality control, we perform repeated analyses of laboratory mineral standards and compare these with results obtained by full-standards WDS analysis on our JEOL-8600 electron microprobe. (We have done a similar set of tests in an evaluation of a Thermo NORAN System $760 \mathrm{~mm}^{2}$ SDD detector.) The results of these tests show that when proper analytical control is maintained, results of comparable precision and accuracy -and in certain cases superior accuracy - can be obtained with the SDD for major and significant minor element concentrations. This is the case even when using the same set of collected standards on the SDD for a several month period, as shown in Table 1.

The most difficult factors in performing high quality SDD analyses on the SEM involve the SEM itself, e.g., beam current stability, accuracy of beam current measurement (or estimation), specimen position relative to the SDD detector 'efocusing' of the detector with position at low magnifications. It is also critical to determine that pulse pile-up is being properly corrected. Most of these factors are easier to control in an electron microprobe. The combination of totally integrated high quality SDD detector(s) with multiple WDS detectors in an electron microprobe make the ideal analytical microbeam analysis instrument of the future [e.g., 2-3].

\section{References:}

[1] N.W.M. Ritchie, D.E. Newbury, and J.M. Davis, Microscopy Microanal. 18 (2012), 892-904.

[2] Armstrong, J. T., P. McSwiggen and C. Nielsen, Microscopy and Analysis 27(7) (2013), 18-22.

[3] Armstrong, J. T., P. McSwiggen and C. Nielsen (2013) Microscopy and Microanal. 19 (Suppl. 2) (2013), 1254-5, doi:10.1017/S143192761300826X. 


\begin{tabular}{|c|c|c|c|c|c|c|c|c|c|c|c|}
\hline JEOLSEM & Oxford & \multirow[t]{2}{*}{ SDD } & \multirow[t]{2}{*}{$15 \mathrm{keV}$} & \multirow[t]{2}{*}{$1 \mathrm{nA}$} & \multirow[t]{2}{*}{ vs } & \multirow[t]{2}{*}{ JEOL 8600} & \multirow[t]{2}{*}{ EPMA } & \multirow[t]{2}{*}{ WDS } & \multirow[t]{2}{*}{$15 \mathrm{keV}$} & \multirow[t]{2}{*}{$30 \mathrm{nA}$} & \\
\hline Basalt Glass & \multirow{2}{*}{$\begin{array}{c}\text { CIW } 30 \\
\text { o }\end{array}$} & & & & & & & & & & \\
\hline & & $\mathrm{Na}$ & $\mathrm{Mg}$ & $\mathrm{Al}$ & $\mathrm{Si}$ & $\mathrm{K}$ & $\mathrm{Ca}$ & $\mathrm{Ti}$ & $\mathrm{Mn}$ & $\mathrm{Fe}$ & Total \\
\hline Nominal & 44.78 & 1.76 & 5.20 & 9.11 & 22.47 & 0.21 & 8.13 & 0.68 & 0.12 & 7.48 & 99.94 \\
\hline WDS $1(n=30)$ & 45.62 & 1.86 & 5.34 & 9.26 & 23.04 & 0.15 & 8.14 & 0.72 & 0.13 & 7.39 & 101.65 \\
\hline Std Dev & 0.29 & 0.003 & 0.08 & 0.05 & 0.16 & 0.01 & 0.07 & 0.03 & 0.004 & 0.08 & 0.62 \\
\hline WDS $2(n=10)$ & 45.35 & 1.86 & 5.25 & 9.22 & 22.93 & 0.16 & 8.11 & 0.70 & 0.13 & 7.32 & 101.03 \\
\hline Std Dev & 0.07 & 0.02 & 0.03 & 0.03 & 0.05 & 0.01 & 0.09 & 0.01 & 0.01 & 0.05 & 0.15 \\
\hline WDS Avg & 45.49 & 1.86 & 5.30 & 9.24 & 22.99 & 0.16 & 8.13 & 0.71 & 0.13 & 7.36 & 101.34 \\
\hline Std Dev & 0.19 & 0.00 & 0.06 & 0.03 & 0.08 & 0.01 & 0.02 & 0.01 & 0.00 & 0.05 & 0.44 \\
\hline $\begin{array}{c}\text { WDS - Nom. } \\
4 / 2013\end{array}$ & 0.70 & 0.10 & 0.09 & 0.13 & 0.52 & -0.06 & -0.01 & 0.03 & 0.01 & -0.13 & 1.40 \\
\hline $\operatorname{SDD}(n=10)$ & 44.70 & 1.77 & 5.20 & 9.15 & 22.37 & 0.16 & 8.13 & 0.70 & 0.13 & 7.63 & 99.94 \\
\hline Std Dev & 0.42 & 0.01 & 0.03 & 0.02 & 0.06 & 0.01 & 0.04 & 0.02 & 0.00 & 0.03 & 0.50 \\
\hline SDD-Nom & -0.08 & 0.01 & 0.00 & 0.04 & -0.10 & -0.05 & 0.00 & 0.02 & 0.01 & 0.15 & 0.00 \\
\hline SDD QC & Std'n of & $5 / 10 / 13$ & & & & & & & & & \\
\hline $5 / 13-2 / 14$ & 0 & $\mathrm{Na}$ & $\mathrm{Mg}$ & $\mathrm{Al}$ & $\mathrm{Si}$ & K & $\mathrm{Ca}$ & $\mathrm{Ti}$ & $\mathrm{Mn}$ & $\mathrm{Fe}$ & Total \\
\hline 1 & 45.03 & 1.73 & 5.15 & 9.00 & 22.57 & 0.16 & 8.20 & 0.68 & 0.14 & 7.47 & 100.13 \\
\hline 2 & 45.06 & 1.75 & 5.25 & 9.12 & 22.50 & 0.17 & 8.32 & 0.71 & 0.16 & 7.60 & 100.64 \\
\hline 3 & 44.44 & 1.71 & 5.14 & 9.05 & 22.10 & 0.15 & 8.24 & 0.70 & 0.17 & 7.53 & 99.23 \\
\hline 4 & 44.38 & 1.66 & 5.19 & 9.19 & 22.28 & 0.16 & 8.24 & 0.69 & 0.09 & 7.72 & 99.60 \\
\hline 5 & 43.98 & 1.73 & 5.13 & 9.32 & 22.20 & 0.16 & 8.27 & 0.70 & 0.14 & 7.45 & 99.08 \\
\hline 6 & 44.48 & 1.73 & 5.20 & 9.25 & 22.37 & 0.16 & 8.18 & 0.70 & 0.16 & 7.68 & 99.91 \\
\hline 7 & 44.37 & 1.74 & 5.21 & 9.36 & 22.60 & 0.18 & 8.34 & 0.69 & & 7.50 & 99.99 \\
\hline 8 & 44.88 & 1.77 & 5.20 & 9.11 & 22.70 & 0.15 & 8.36 & 0.74 & 0.16 & 7.64 & 100.71 \\
\hline 9 & 44.74 & 1.75 & 5.22 & 9.27 & 22.81 & 0.16 & 8.42 & 0.75 & & 7.65 & 100.77 \\
\hline 19 & 43.98 & 1.71 & 5.21 & 9.17 & 22.63 & 0.15 & 8.38 & 0.71 & & 7.55 & 99.49 \\
\hline 11 & 44.74 & 1.69 & 5.22 & 9.30 & 22.54 & 0.15 & 8.30 & 0.73 & 0.12 & 7.53 & 100.32 \\
\hline 12 & 44.36 & 1.69 & 5.22 & 9.30 & 22.45 & 0.14 & 8.40 & 0.69 & 0.17 & 7.56 & 99.98 \\
\hline Average & 44.54 & 1.72 & 5.20 & 9.20 & 22.48 & 0.16 & 8.30 & 0.71 & 0.15 & 7.57 & 99.99 \\
\hline StDev & 0.36 & 0.03 & 0.04 & 0.11 & 0.21 & 0.01 & 0.08 & 0.02 & 0.03 & 0.09 & 0.56 \\
\hline$\%$ RSD & $0.8 \%$ & $1.8 \%$ & $0.7 \%$ & $1.2 \%$ & $0.9 \%$ & & $1.0 \%$ & & & $1.1 \%$ & $0.6 \%$ \\
\hline SD-CntStat & 0.15 & 0.03 & 0.04 & 0.04 & 0.06 & 0.02 & 0.05 & 0.03 & 0.04 & 0.09 & 0.21 \\
\hline Meas - Nom. & -0.24 & -0.04 & -0.01 & 0.09 & 0.01 & -0.05 & 0.17 & 0.03 & 0.03 & 0.09 & 0.05 \\
\hline Oxford & & & & & & & & & & & \\
\hline Factory 'Std' & 46.31 & 2.06 & 4.96 & 8.91 & 20.93 & 0.14 & 8.20 & 0.59 & 0.13 & 6.45 & 98.68 \\
\hline 'Std-less' & 46.93 & 2.09 & 5.03 & 9.03 & 21.21 & 0.14 & 8.31 & 0.60 & 0.13 & 6.54 & 100.00 \\
\hline
\end{tabular}

Table 1. Measured composition of CIW basalt glass standard 30 made by WDS on an electron microprobe and by SDD-EDS on an analytical SEM. Simple mineral standards were used on both instruments. The standards for EPMA-WDS were measured at each analytical session. Those for SDD-EDS were measured only once during the ten month period the measurements were made, Oxygen was determined by stoichiometry for WDS and measured for SDD-EDS. Analyses at bottom in blue are for a typical SDD-EDS analysis when processed through the manufacturer's factory-standards-based 'standardless' analysis, unnormalized and normalized. Standard measurement conditions for the SDD: 50 second counting time at an amplifier setting [PT4] resulting in an input of $\sim 50,000 \mathrm{cps}$ full spectrum and an output of $\sim 30,000-35,000$ cps. 